\title{
Modos de construcción social de sentido en el coro: Timing expresivo entre coreutas y director
}

\section{Artículo de investigación}

\section{Manuel Alejandro Ordás}

Universidad Nacional de La Plata, Argentina ordasalejandro@fba.unlp.edu.ar

Recibido: 9 de abril de 2021

Aceptado: 2 de agosto de 2021

Como citar este artículo: Ordás, Manuel Alejandro. (2022). Modos de construcción social de sentido en el coro: Timing expresivo entre coreutas y director. Calle14: revista de investigación en el campo del arte. 17(31), pp. 50-65.

DOI: https://doi.org/10.14483/21450706.18690

\section{Agradecimientos}

Esta investigación fue posible gracias al soporte y financiamiento de la Secretaría de Ciencia y Técnica de la Universidad Nacional de la Plata (PRD Res.929/19, enmarcado en el Proyecto B349). Asimismo, se agradece a los evaluadores por sus aportes y comentarios para la revisión del artículo.

\section{(c) (1)}

https://creativecommons.org/licenses/by/4.0/deed.es 

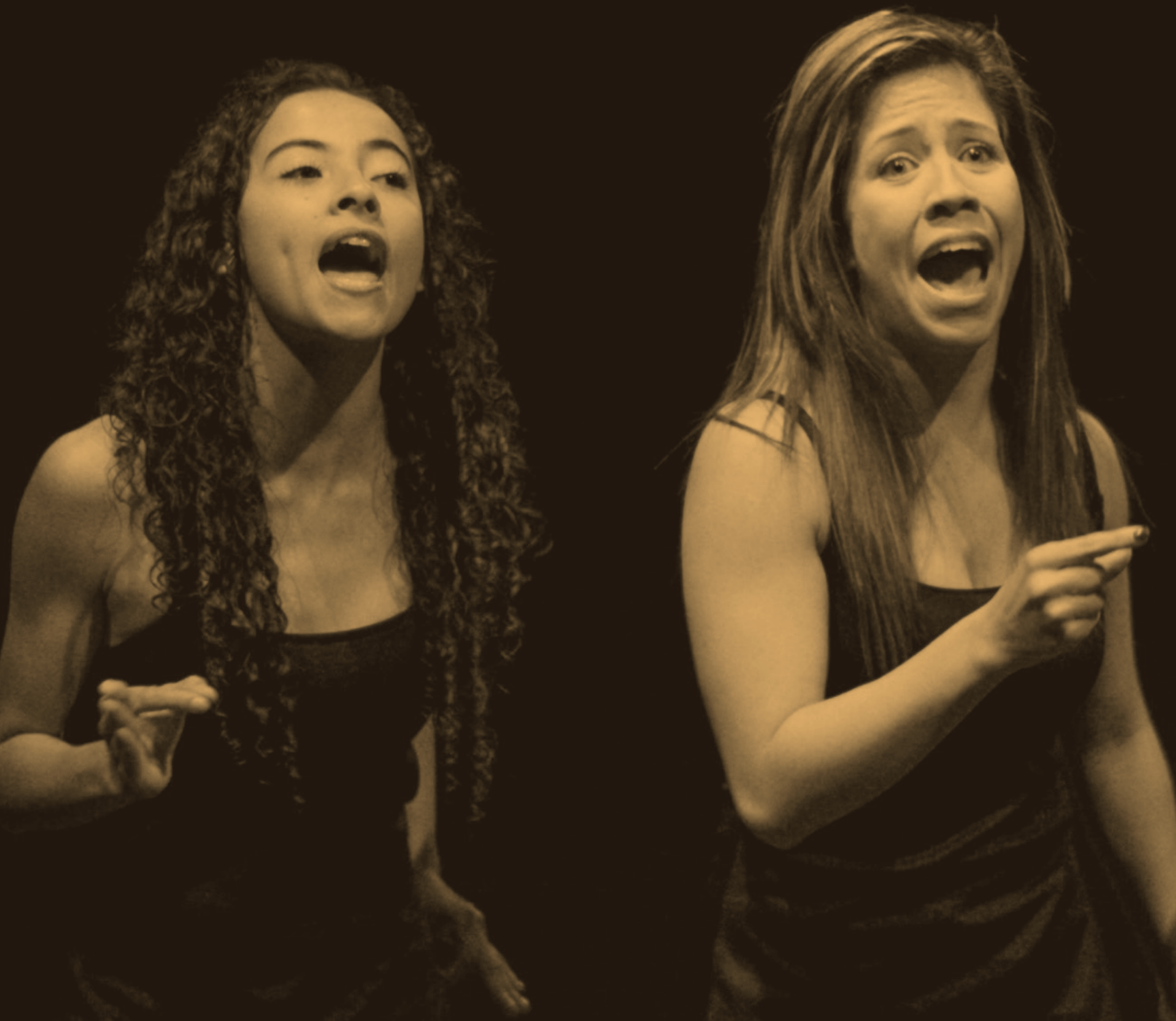
Modos de construcción social de sentido en un coro: Timing expresivo entre coreutas y director

\begin{abstract}
Resumen
Desde una perspectiva interactiva, un coro puede verse como un conjunto de individuos que están en interacción y no como un grupo uniforme subordinado al director. Describimos aquí las interacciones temporales entre cantantes y director durante la ejecución coral mediante un análisis multimodal, para indagar en la variabilidad como un medio de construcción de la interpretación. Los resultados muestran que estar juntos en el tiempo no es producto de una relación unidireccional en la que el director realiza una acción y el coro responde, sino que la sincronía de lo que se canta y la interacción con quien conduce son formas de entonamiento corporeizado. Discutimos la variabilidad en términos de rasgos de diferenciación-indiferenciación individual y grupal que están enraizados en la concepción de la práctica social en el coro.
\end{abstract}

\title{
Palabras clave
}

Análisis multimodal; práctica coral; timing expresivo; variabilidad temporal

Modes of social construction of meaning in a choir: Expressive timing between choir singers and director

\begin{abstract}
From an interactive perspective, a choir can be understood as a set of individuals who are interacting and not as a uniform group that is subordinate to a director. We aim to describe the temporal interactions between singers and director during a choral performance by means of a multimodal analysis, in order to inquire in variability as a means of construction of the performance. The results showed that being together in time is not the product of a unidirectional relationship where the director performs an action and the choir responds, but rather that the synchronicity of what is sung and the interaction with the director are forms of embodied intonation. We discuss variability in terms of individual and group differentiationundifferentiation traits that are deeply rooted in the conception of social practice in the choir.
\end{abstract}

\section{Keywords}

Multimodal analysis; choral practice; expressive timing; temporal variability

Modes de construction sociale du sens dans un chceur : timing expressif entre choristes et chef de choeur

\section{Résumé}

D'un point de vue interactif, un chœur peut être compris comme un ensemble d'individus en interaction et non comme un groupe uniforme subordonné à un chef de chœur. Nous visons à décrire les interactions temporelles entre chanteurs et chef de chœur lors d'une performance chorale au moyen d'une analyse multimodale, pour enquêter sur la variabilité comme moyen de construction de l'interprétation. Les résultats ont montré qu'être ensemble dans le temps n'est pas le résultat d'une relation unidirectionnelle où le chef de chœur exécute une action et le chœur répond, mais plutôt que la synchronicité de ce qui est chanté et l'interaction avec le chef de chœur sont des formes d'intonation incarnée. Nous discutons de la variabilité en termes de traits de différenciation-indifférenciation individuels et collectifs si profondément enracinés dans la conception de la pratique sociale dans le chœur.

\section{Mots clés}

Analyse multimodale ; pratique chorale ; timing expressif ; variabilité temporelle 


\section{Resumo}

A partir de uma perspectiva interativa, se entende o coro como um conjunto de indivíduos que estão em interação e não como um grupo uniforme subordinado ao diretor. Neste trabalho, descrevemos as interações temporais entre cantantes e diretor durante a execução coral mediante uma análise multimodal, para indagar a variabilidade como um meio de construção da interpretação. Os resultados mostraram que estar juntos no tempo não é produto de uma relação unidirecional, na qual o diretor realiza uma ação e o coro responde, senão que, a sincronia do que se canta e a interação com quem conduz são formas de entonação corporeizadas. Discutimos a variabilidade em termos de características de diferenciação-indiferenciação individual e grupal tão enraizadas na concepção da prática social no coro.

\section{Palavras chave}

análise multimodal; prática coral; timing expressivo; variabilidade temporal

\section{Rrurai kallariska tukuikunaua iuiarispa tunangapa allilla rupaikuna Iachachig iachaikugkunaua}

\section{Maillallachiska}

Allilla kawaspa Cora ninallami Achka rurakuna chillatata ruradurkuna suglla wachu katichingapa, kaipi katichiku kilkaspa tutadurkunawa Sugllapi tukuspa Sugllapi tukuchingapa, chasa ullarinchi sugpi sugpi kawangapa, kawachingapa sapalla chasalla tukuikuna uramanda kaitaringapa ikuti tukuikunawa.

\section{Rimangapa Ministidukuna}

Tapuchispa imasa ruraikuna; iachaikui tunangapa; allilla ruraikuna kawachii; suura chukarri 


\section{Introducción}

Todo director, particularmente los directores de los coros vocacionales, sabemos que, si no trabajamos sobre la construcción de la variabilidad, nunca vamos a acercarnos al ideal sonoro estandarizado desde el modelo tradicional de la cuerda como una voz única, homogénea. Pero, en efecto, cuando consideramos esta variabilidad, es decir, cuando la sentimos y la percibimos, no sabemos exactamente de qué está compuesta. Tampoco sabemos qué aspectos de la variabilidad de la ejecución vocal podrían relacionarse, por lo tanto; tenemos que trabajar auditivamente modelando el sonido, probablemente en términos de una co-construcción junto con los coreutas. Entendemos a la construcción del sonido coral como un aspecto crucial para la práctica de un coro vocacional, sin embargo, no existe una investigación sistemática que aborde este tema.

Tradicionalmente, la variabilidad vocal dentro del coro (o la variabilidad percibida dentro de las cuerdas) ha sido considerada principalmente como el resultado de errores en la ejecución. Sin embargo, podría ser útil considerarla de una manera bastante diferente, y pasar a comprenderla como resultados internos diversos, con los que el director tiene que trabajar para lograr niveles de homogeneidad crecientes. En el transcurso de esta investigación pretendemos atender y tener en cuenta esta diversidad heterogénea que forma parte de la práctica.

En el presente estudio, hemos analizado datos de una investigación más amplia que puso el foco en estudiar la comunicación entre el director y sus coreutas, para identificar comportamientos particulares que denotan variabilidad en la actuación de un coro amateur (Ordás, 2017). La variabilidad temporal es una característica central de la interpretación musical. En el caso de la música coral, las diferentes frases de la obra se interpretan de forma multivocal en el tiempo. Proponemos que la variabilidad es lo que caracteriza la actividad interna de la interpretación cantada de la obra coral, o, en otras palabras, lo que es aparentemente homogéneo, internamente resulta ser bastante variable.

El timing expresivo, a través de la coordinación temporal y la expresividad, es un componente esencial de la comunicación entre el director y el coro. Ha sido estudiado tanto desde la perspectiva del director (Damesón, 2018; Ordás y Martínez, 2019a) y del coreuta dentro de la cuerda (Ordás y Martínez, 2017; Ordás, 2018) por ejemplo, en términos de parámetros físicos de los gestos de dirección (Ordás, Martínez, y Alimenti Bel, 2014), como desde la perspectiva del observador (Ordás y Martínez, 2013), por ejemplo, en términos de evaluación de la expresividad musical comunicada (Ordás y Blanco Fernández, 2013). Sin embargo, es necesario comprender mejor el rol de los diferentes factores que influyen en la comunicación del director sobre el timing expresivo. Estudios recientes (Pazniakova et al., 2016) investigaron cuatro factores que influyen en la sensibilidad al timing expresivo comunicado por un director: (i) la experiencia de actuar bajo la conducción de un director, (ii) la capacidad de moverse junto con el director, (iii) el papel de las modalidades visuales y auditivas, y (iv) el tempo del timing expresivo. Los resultados revelaron que la comunicación del director sobre el timing expresivo depende de una interconexión de experiencias, los tempi y las modalidades del beat o pulso. Por otro lado, la capacidad de moverse junto con el director no mejoró significativamente la performance del timing expresivo de los participantes, lo que requiere investigación adicional.

El tema de la interacción, la comunicación y la regulación temporal entre intérpretes ha sido de interés en el campo de la cognición musical y el estudio de la performance. Un estudio sobre dúos y solos de piano (Loehr y Palmer, 2011) examinó, si las representaciones de las acciones de los músicos se activan en los co-intérpretes con los que se deben coordinar acciones en el tiempo, y si los co-intérpretes simulan las acciones de los demás utilizando sus propios sistemas motores durante la coordinación temporal. Se encontró que los músicos que tenían ritmos similares en la interpretación solista, estaban mejor sincronizados y mostraban una adaptación mutua a la sincronización del otro durante la performance a dúo. También se ha investigado la coordinación temporal en un cuarteto de cuerdas (Timmers et al., 2014). Allí se identificaron patrones temporales del tipo orientador-orientado: la conducta de anticipación del primer violín precedía microtemporalmente a los ataques del resto de los músicos. Por otro lado, en el ámbito del jazz, por mencionar otro estilo musical, se examinaron los efectos de la variabilidad temporal horizontal y vertical del beat entre el saxofón, el bajo y la batería (Wesolowski, 2016): se observó que la sincronía temporal entre los músicos se vinculaba además con otras variables musicales como el carácter melódico de los pasajes, la armonía subyacente y los tempi entre secciones. Otros estudios exploraron la interactividad en la improvisación en tríos de jazz como una forma de construcción participativa de sentido

(Martínez et al., 2017). 
Se encontró que la actividad de movimiento de dos músicos saxofonistas improvisando junto a la base de un pianista, se produce no sólo cuando tocan sino cuando esperan el turno para tocar, resultando, en este último caso, en un movimiento congruente con las características de la producción sonora del saxofón a cargo de la ejecución. Por otro lado, comprobaron cómo la producción sonoro-kinética de un saxofón influía en la del otro saxofón, lo cual evidencia la actividad comunicativa en la construcción musical conjunta. Algunos estudios en la misma línea (Schiavio, 2014; Schiavio y De Jaegher, 2017) sostienen que la práctica musical conjunta se comprende mejor cuando se considera a los intérpretes como agentes autónomos e interactivos que negocian en tiempo real sus emociones, al igual que las habilidades sensoriomotoras y comunicativas. El estudio de la comunicación entre músicos bajo la teoría de los sistemas dinámicos hace énfasis en el concepto de entrainment y lo utiliza para estudiar los mecanismos de regulación temporal, esto es, la posibilidad de estar en fase o fuera de fase con otro u otros durante la práctica de la ejecución musical (Clayton, 2012; 2013; Clayton, Sager, y Will, 2005).

Desde una mirada corporeizada e intersubjetiva, la comunicación expresiva en la práctica musical basa su producción de sentido en la interacción dinámica de los cuerpos en acción de los intérpretes, y no exclusivamente en procesos mentales individuales de atribución de sentido (Schiavio y Høffding, 2015). Así, podría indagarse el movimiento corporal de los intérpretes en el coro (coreutas y director) haciendo hincapié en los cambios y continuidades que tienen lugar durante la construcción intersubjetiva, sea que la misma corresponda a la elaboración de la interpretación de una obra en estilo, o que se trate de la elaboración de música en tiempo real, como sería el caso, por ejemplo, de la improvisación en un estándar de jazz (Martínez et al., 2017).

Ahora bien, ¿cómo podríamos observar todo esto durante la performance coral? Para intentar brindar una respuesta, seleccionamos algunas instancias de una ejecución para analizar cómo se manifiesta la variabilidad en la práctica de un coro vocacional, cuáles son sus posibles descriptores y cuáles son sus causas probables, mediante un análisis multimodal de sonido y movimiento que vincula dos dimensiones: (i) el timing intra-cuerda, en nuestro ejemplo tomamos las cuerdas de sopranos y bajos con tenores al unísono; y (ii) la relación entre la producción sonora y los movimientos del director de coro.
Por todo ello, nos proponemos avanzar hacia una explicación con base en la identificación de la variabilidad temporal que subyace en una práctica coral amateur a través del análisis multimodal.

\section{Método}

\section{Participantes}

Quince coreutas de un coro vocacional $(n=15)$ fueron videograbados durante el ensayo de una performance coral, quienes participaron voluntariamente para este estudio. El coro estuvo conformado por diez mujeres, y cinco hombres con un promedio de edad de 49,1 (sd = 14,6), cuyos integrantes contaban con una experiencia coral promedio de seis años. La distribución según su clasificación vocal fue de cinco sopranos, cinco contraltos/mezzosopranos, un tenor, y cuatro barítonos/bajos.

\section{Caracterización de la población - coro participante}

El coro participante del estudio funcionó durante 8 años como una agrupación institucional estable del Hospital El Cruce-Alta Complejidad en Red Dr. Néstor Carlos Kirchner en Florencio Varela, Buenos Aires, Argentina. Una selección de personas de este grupo fue la que participó activamente del presente estudio. El coro nació a raíz de la voluntad institucional, y de su director, de conformar una actividad cultural estable que represente al hospital y mediante su actividad artística, llegar a la comunidad. En principio pensado para estar conformado por todos aquellos que formen parte del hospital, en cualquiera de los servicios, pero rápidamente ampliado a todos aquellos habitantes de la zona que quisieran acercarse a realizar la actividad, bajo el lema de todos podemos cantar.

Suponiendo que una institución dedicada a brindar salud de alta complejidad ayuda a mejorar la vida de las personas, se piensa también en ofrecer a la comunidad la puesta en funcionamiento de un coro, siendo la música pensada como un componente de práctica social enriquecedor, y entendiendo el arte en general como parte fundamental de la sociedad dado que, a raíz de esta propuesta, se organizaron grupos de teatro, también vinculados al hospital. Encontramos en la actividad de un coro, que su manifestación mediante el canto complementa de alguna manera las necesidades institucionales de brindar los servicios de salud comunitarios que se proveen. 
De este modo, nació el coro, como un grupo de nivel vocacional o amateur, con un repertorio en su mayoría compuesto por música popular urbana argentina y latinoamericana. En toda su historia, estuvo conformado por cantantes jóvenes y adultos en un rango desde 18 años hasta los 69 años (media =46,8), con la inclusión de algunos trabajadores de distintas áreas del mismo hospital. El resto de los integrantes, provenían de las zonas aledañas que conforman el Gran Buenos Aires, tales como las comunidades de Florencio Varela, Quilmes, Berazategui, Ranelagh, San Francisco Solano y demás comunas cercanas. Las personas que integraron el grupo provenían de ambientes muy diversos a las que las unió sólo el gusto por cantar, donde ninguno se dedicaba a la música profesionalmente, sino que desarrollaban diversas profesiones y ocupaciones en su vida cotidiana. Tal es así que hubo docentes, profesores, jubilados/as, empleados/as públicos, administrativos/ as, profesionales de la salud y hasta jóvenes estudiantes universitarios de diversas carreras. Si bien no fue excluyente el saber musical o la experiencia coral para ingresar, a las personas que manifestaban su voluntad de cantar en el coro, se les realizaba una audición previa a los efectos de evaluar su voz para obtener un diagnóstico vocal general, principalmente enfocado en el aspecto registral y la salud vocal.

Las clasificaciones vocales se dividieron mayormente en los cuatro registros típicos de la mayoría de los coros vocacionales mixtos, asociados a las cuerdas de sopranos, mezzosopranos/contraltos, tenores, y barítonos/ bajos. El grupo se juntaba dos veces por semana, en ensayos de dos horas, en el mismo hospital. Si bien en los comienzos de su actividad los ensayos eran sólo una vez por semana, el carácter vocacional del grupo y las ganas de mejorar, sumado al interés y al compromiso de la institución con sostener la actividad, se incrementó al doble la carga horaria. Esto impactó de manera positiva en el rendimiento musical del grupo, profundizando las relaciones interpersonales, y sumando momentos compartidos en el desarrollo de la actividad, como por ejemplo viajar a festivales corales. Los ensayos constaban de un perfilamiento del trabajo vocal (respiración, autoconciencia corporal, posturas, emisión vocal, desarrollo auditivo-perceptivo) y la preparación de las obras de repertorio habitual del coro para su interpretación. Desde la dirección del coro, estos aspectos implican además el desarrollo de la coordinación gestual y la construcción creciente de los criterios de conducción musical que la realización de cada obra demanda. Esto, trazando una analogía con la actividad de un escultor, se podría comprender como una forma de ir esculpiendo, en este caso, el sonido, para llegar a una construcción del sonido conjunto. Durante el desarrollo de la actividad y la práctica, los participantes fueron adquiriendo las nociones técnicas del canto coral conjunto, y aunque todos los años se sufre un movimiento inevitable de gente que abandona y gente que ingresa al grupo, se mantiene una base estable de cantantes que permite el crecimiento sostenido del coro.

El contexto de actuación de este coro es variado y bastante amplio en relación a su actividad artística. Participó en gran cantidad de conciertos, encuentros junto a otros coros, festivales y ciclos corales periódicos, tanto en su zona de influencia (zona sur del Gran Buenos Aires) como en la Ciudad Autónoma de Buenos, Aires y varias provincias argentinas. Su intensa actividad musical incluía además la organización de un festival coral internacional, el Festival Coral Balcarce Canto y Raíces donde recibían en la ciudad de Balcarce (Provincia de Buenos Aires) a otros coros de todo el país, e incluso del exterior. También realizaba muchos conciertos didácticos en escuelas, bibliotecas populares, y en diversos eventos culturales, tales como ferias del libro, por mencionar sólo alguna. Dentro de su actividad artística, el coro también realizaba producciones corales y/o sinfónico-corales independientes por encargo. En su comunidad, se constituyó como una asociación civil sin fines de lucro para profundizar la difusión de la actividad coral en su medio, lo que llevó a que recibieran el reconocimiento como entidad de bien público por parte del Municipio de Florencio Varela, Buenos Aires, Argentina.

La selección del repertorio que abordaba el coro, en la mayoría de los casos quedaba a criterio de su director, aunque a menudo, los cantantes tenían la libertad de sugerir la realización de distintas obras de su agrado al momento de ir ampliando el repertorio, lo cual muchas veces fue aceptado y muy bien recibido por el director, siempre y cuando la obra en cuestión se adaptare a las características musicales del grupo. La conformación del repertorio resulta de inevitable incumbencia con la programación anual y la eventual inclusión de las obras propuestas por los coreutas configuran un incentivo para los cantantes, al tratarse de obras cuya interpretación les resulta interesante, novedosa, y/o desafiante desde algún punto de vista. Acorde a nuestra idea de comprender al coro como un organismo vivo e interactivo es importante saber escuchar, y no meramente en el sentido acústico de la palabra, sino atender a lo que el coro dice, reconociendo a su vez las individualidades, ya que muchas veces el coro no habla como 
un único organismo, como un todo uniforme, sino que hay muchas voluntades y subjetividades (a veces hasta enfrentadas) en un pedido o sugerencia, y el director debería poder saber leer también esas individualidades, unificarlas, y guiarlas hacía una única intención o idea.

En la actualidad, el estado del arte del estudio de la música y su experiencia es multidisciplinario e invita a un pensamiento vincular-relacional no admitiendo el pensamiento reduccionista. Por lo tanto, ubicar el caso de estudio en un grupo conocido por el autor, provee un ambiente rico para estudiar las problemáticas de la comunicación en el coro, al incluir una cosmovisión plena de la actividad, de su inserción cultural, social e institucional, tanto del grupo coral como de los integrantes. Conocer a las personas que participan, sus intenciones, deseos, percepciones y hasta limitaciones posibilita dar cuenta de muchos aspectos silenciados en las relaciones de ensambles dirigidos, poniendo el foco en la perspectiva del coreuta. Tomar una visión íntegramente de tercera persona, como investigador externo de un hecho artístico, por el contrario, brindaría una visión diferente del problema, dado que quedaría fuera del estudio un cúmulo de información relevante (como considerar la experiencia y la formación) que puede ser útil para la construcción de la discusión. Por otro lado, en términos del estudio de la comunicación, el tomar como objeto de investigación la actividad de un coro vocacional provee una riqueza de interacciones que forman parte de la práctica, puesto que, el trabajo con este tipo de coros demanda un esfuerzo comunicativo mayor por parte del director para suplir algunas limitantes o habilidades faltantes en términos de lectura musical, comprensión estilística, código gestual estandarizado de dirección, autoconocimiento de las potencialidades vocales, y demás.
De algún modo nos cuestionamos acerca del componente individual frente al grupal. En este sentido, el modelo cultural tradicional intenta eliminar la individualidad como parte de la construcción de significado en la práctica del coro.

\section{Estimulo}

Se seleccionó un arreglo coral de Diego Sarquis de la obra De música ligera, con música de Gustavo Cerati, del cual se eligió para el análisis un fragmento que comprende 19 compases de la re-exposición del tema. Se trata de una obra que posee cambios de carácter entre las frases, expresada en cambios de tempo, y con una textura contrapuntística tanto imitativa como homorrítmica en el tratamiento de las voces hacia el interior de las frases.

\section{Caracterización del estímulo}

El fragmento objeto de análisis representa un punto de articulación formal relevante dentro de la obra: se sitúa en la segunda parte de la misma, con lo cual ya se cantó el primer texto, y si bien el fragmento en cuestión presenta una repetición de las frases, esta vez se cantan con un texto diferente sumando un cambio de cierre hacia el final (es decir, se configura la misma estructura, pero con un distinto significado semántico). Entendemos que todo este fragmento funcionaría como una preparación para la gran articulación formal a donde se llega, previo a una coda para finalizar la obra (Figura 1).

Los 19 compases del fragmento seleccionado para el análisis presentan una estructura métrica en 4/4 y consta de dos grandes secciones que podemos dividir entre estrofa y estribillo con una suerte de coda de 3 compases al final de la sección. En la sección a el texto pertenece a las voces masculinas quienes cantan la

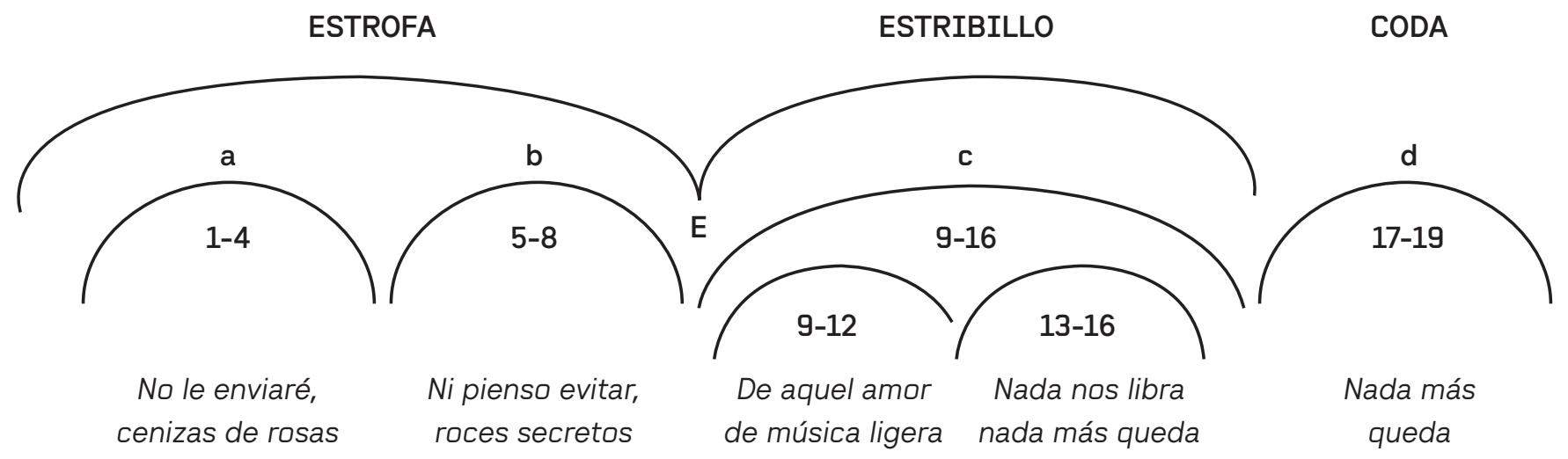

Figura 1. Estructura formal de los 19 compases del fragmento analizado del estímulo. Los números de los arcos corresponden a los compases que dura cada sección con el texto correspondiente. 
melodía principal al unísono (no le enviaré, cenizas de rosas), y las voces femeninas cantan un acompañamiento duplicado empleando sonidos de onomatopeyas, que refuerzan el aspecto rítmico del rol musical que les toca ejecutar (dum-dum du-rúm, dum). Los hombres continúan con la siguiente frase (sección b) sobre el texto ni pienso evitar, con una imitación rítmica complementaria de las mujeres que se suceden una atrás de la otra (contraltos-sopranos), y completan esa frase con el texto roces secretos. Luego las voces femeninas inician el nuevo texto de aquel amor, anticipadamente, como una preparación o anacrusa al estribillo (E) en imitación con las voces masculinas que finalmente llegan al estribillo dando comienzo a la sección c (en el compás 9). La sección c comprende los compases 9 a 16 dentro de los cuales podemos identificar dos frases con el texto de aquel amor, de música ligera (compases 9 a 12) y nada nos libra, nada más queda (compases 13 a 16) las cuales guardan internamente el mismo tratamiento contrapuntístico: el tenor lleva la melodía principal, duplicada en el bajo, mientras que se sucede una imitación del motivo rítmico en sopranos y luego en las contraltos (a una distancia de dos tactus entre sí). La última sección $(d)$ que finaliza el fragmento analizado (compases 17 a 19) se caracteriza por repetir el texto nada más de manera imitativa desde el tenor a la soprano, pasando por las contraltos también con una distancia de dos tactus. Finalmente, todas las voces se juntan en homorritmia con los bajos (al final del compás 18) dando lugar a un retardo, acompañado de un gran rallentando que detiene el movimiento de la música para que las contraltos resuelvan la frase.

\section{Aparatos}

Los registros globales e individuales de audio se realizaron con 16 Micrófonos Shure SM57, una consola de Audio Yamaha 01v96, interface de Audio M-Audio ProFire2626, el software de grabación Logic Pro y un grabador de audio ambiente Zoom H4. El pre-procesamiento de los archivos de audio implicó la sincronización de archivos, el filtrado básico y la limpieza mediante el software Reaper 5.211 y Sonic Visualiser 2.5 (Cannam, Landone, y Sandler, 2010). El procesamiento y la organización posterior del conjunto de datos se realizaron utilizando herramientas de detección del pulso Aubio Beat Tracker (Vamp Aubio Plugins) y la planilla de cálculos en Microsoft Excel 2010 (Office Suite). La captura y análisis de los datos de movimiento del director -tomados de la grabación de vídeo en 2D- se realizó mediante el software Tracker.

\section{Diseño y procedimiento}

Se realizó un registro audiovisual de la performance del coro vocacional objeto de estudio simulando un ambiente de concierto en el lugar de su ensayo habitual. Se utilizaron micrófonos individuales para obtener la voz de cada participante en pistas de audio por separado. Para el análisis de la temporalidad intra-cuerda, se obtuvieron los perfiles de desvíos de timing de cada una de las voces mediante una anotación sobre la señal sonora. El perfil de timing de la ejecución de cada participante del coro se superpuso a los perfiles de movimiento del director.

\section{Resultados}

En primer lugar, hay que decir que el resultado de la audición del coro objeto de estudio es coherente con un grado de homogeneidad bastante aceptable. Por lo tanto, no toda la diversidad debe interpretarse como cantar mal. Por lo tanto, al analizar la variabilidad interna esperamos identificar algunas situaciones interesantes como las que vamos a describir a continuación.

\section{Análisis del timing intra-cuerda}

Las mayores desviaciones de timing en el comportamiento de la cuerda de sopranos se encuentran en los onsets ${ }^{1} 19-20$ y 66-67 (Figura 2). Musicalmente hablando, hay valores largos en o.19 y valores cortos en o.66; ambos casos representan una inercia de alguna regularidad temporal que es variada o modificada instantáneamente. Así, esta ruptura de la isocronía dentro de la textura coral puede deberse a un comportamiento adaptativo entre la voz soprano y las otras voces; es decir, la variabilidad temporal responde a una cierta adaptación con lo que la otra voz está cantando (precedente y posteriormente).

En la Figura 2 podemos observar: (i) punto de llegada/ culminación de la frase (sopranos o.19-20) frente al comienzo de la nueva frase musical con un levare de corchea (varones 0.20); y (ii) sucesivas entradas sobre el texto "nada más" del tenor, la contralto, y finalmente la soprano quienes terminan en un gran rallentandi.

Entre los 0.41 y 47 encontramos mucha homogeneidad. Los cantantes van todos juntos produciendo valores más

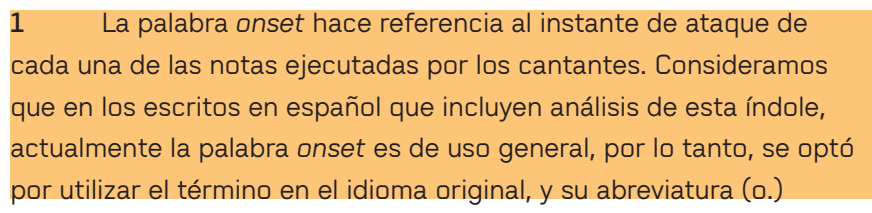




\section{Desvíos de timing (SOPRANOS)}

600,000

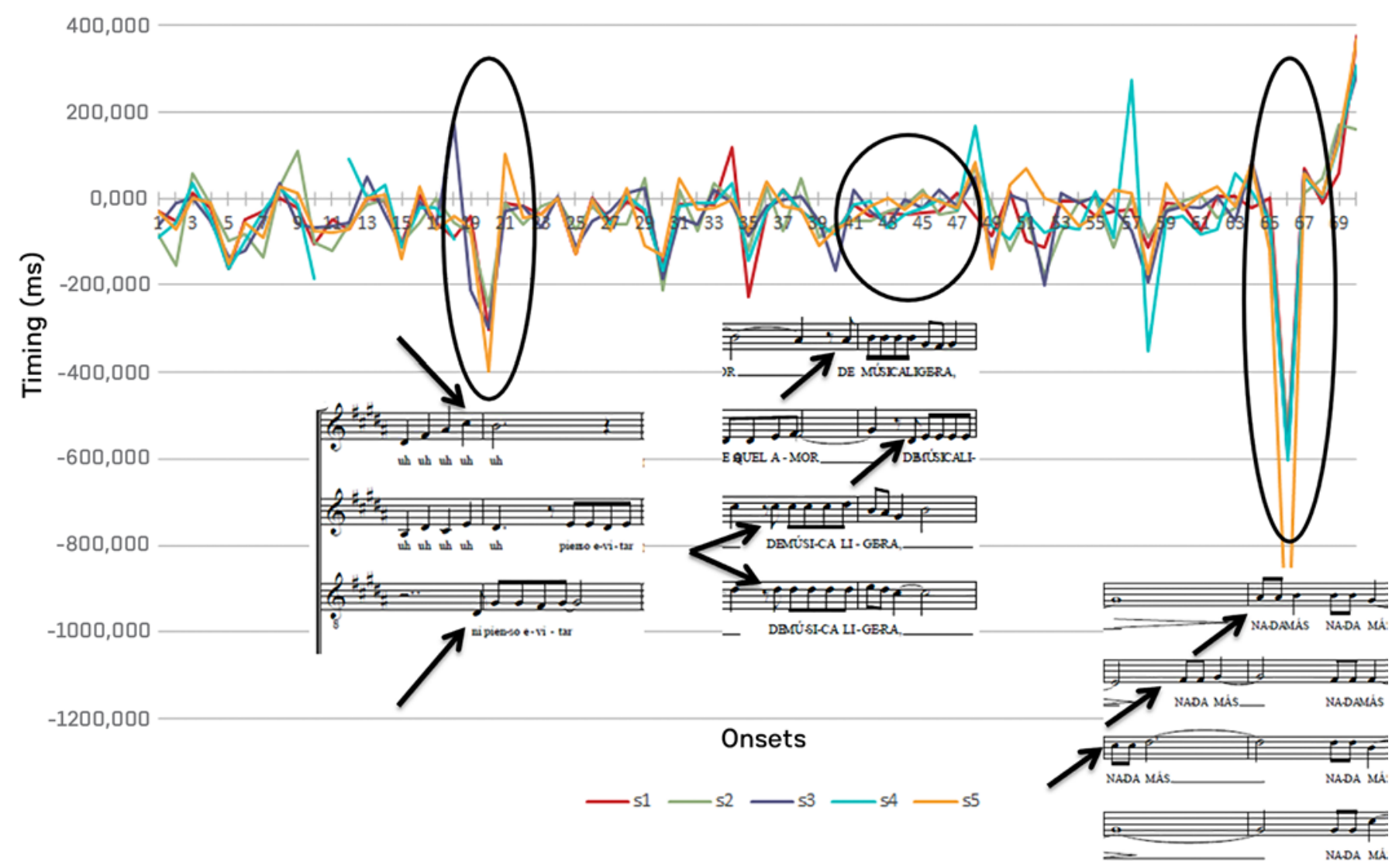

Figura 2. Perfiles de timing de la cuerda de sopranos. El eje y muestra los valores de desvíos temporales en milisegundos. El eje x corresponde a las notas cantadas por cada soprano.

cortos y articulados. Musicalmente hablando, en términos de contrapunto, aquí se sucede una imitación con el resto de las voces sobre el texto "de-música" (hombres con melodía duplicada); "de-música" (sopranos), "de-música” (altos, imitación a un intervalo de 5ta.).

En la Figura 3, observamos aceleraciones y deceleraciones que parecieran compensaciones temporales de los cantantes dentro de la cuerda:

En este caso, todos los desvíos temporales son retrasos (expresados en los valores positivos) que luego se compensan con una anticipación (expresado en los valores negativos). Identificamos los siguientes lugares temporales de acuerdo al instante de ataque u onset, en relación al número de cantante-soprano y la desviación de timing registrada (ver Figura 3): o.9-10 sobre s2, o.18-19 en s3, o.34-35 en s1 (esta soprano es la voz líder de la cuerda, la que está más adelantada; aquí observamos un ejemplo de la compensación más clara), o.48-49 en s3 y s5, y o.57-58 en s4.

Los estudios de timing siempre hablan de compensación. Las aceleraciones y desaceleraciones también se compensan para proporcionar una regularidad temporal general de una interpretación determinada, es decir, mantener el tempo de la performance. En el caso de un coro, es el director quien conduce esta temporalidad general. En este sentido, la variabilidad temporal, a pesar del gesto estricto del director, se regiría por la ley de las compensaciones, como ya se investigado en los estudios clásicos acerca de la temporalidad en la performance (Repp, 2000; 2002; 2005; Repp y Doggett, 2007; Repp y Keller, 2004; Repp y Penel, 2002; Repp y $\mathrm{Su}, 2013)$. La compensación pareciera ser una característica humana adaptativa del sistema dinámico de la performance, y así, parece ser el comportamiento general (a la vez que variable) del coro. Mientras todos 


\section{Desvíos de timing (SOPRANOS)}

600,000

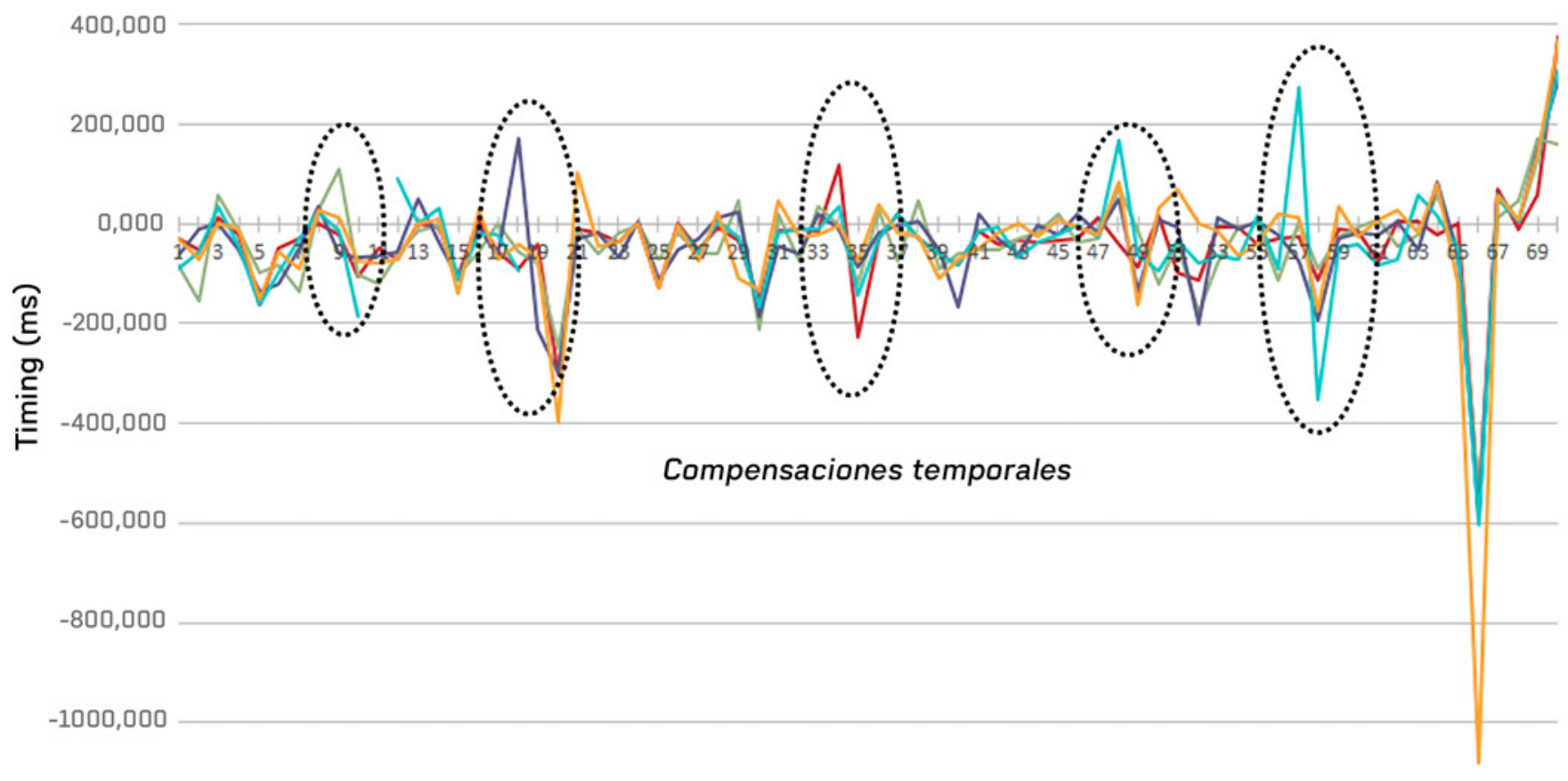

$-1200,000$

Onsets

$-s 1-s 2-s 3-s 4-s 5$

Figura 3. Perfiles de timing de la cuerda de sopranos. Los círculos punteados indican las compensaciones temporales.

cantan juntos un cierto ritmo, dentro de las cuerdas del coro, incluso los cantantes menos aptos se rigen por la ley de la compensación (por ejemplo, en los lugares temporales analizados en la Figura 3).

Las aceleraciones y desaceleraciones también se compensan para proporcionar una regularidad temporal general de una interpretación determinada, es decir, mantener el tempo de la performance. En el caso de un coro, es el director quien conduce esta temporalidad general. Sin embargo, dentro de esta tendencia general, en este caso observamos que los retrasos se compensaron inmediatamente.

\section{Análisis de los movimientos del director y la producción sonora de coro}

Realizamos un análisis para determinar cuál es el rol que cumplen los movimientos del director en este caso, en relación a la ejecución interactiva de los coreutas. La Figura 4 muestra una segmentación de la suma de los movimientos del director para los onsets de sopranos y el unísono de tenores y bajos en el mismo lugar temporal de la música. Establecimos los marcadores de trackeo —o seguimiento- para capturar el movimiento en la cabeza, ambas manos y ambos hombros del director. La curva azul superior es un movimiento que no está en la misma escala que las desviaciones, que es una escala de tiempo. Se hizo una verificación para una comparación. No es necesario centrarse en la escala del movimiento, ya que no tiene mucho significado, pero indica la dinámica de los cambios de movimiento (y la cantidad de movimiento).

Desde esta ubicación específica (en sopranos y tenores-bajos) seleccionada para el análisis, el director marca la corchea a los varones con la cabeza y luego hace un gesto con un cambio repentino de velocidad y trayectoria (de arriba hacia abajo) de las manos. Este gesto se sitúa en el o.19 y 20 de las sopranos (o.10 y 11 de los varones respectivamente). Las sopranos llegan 


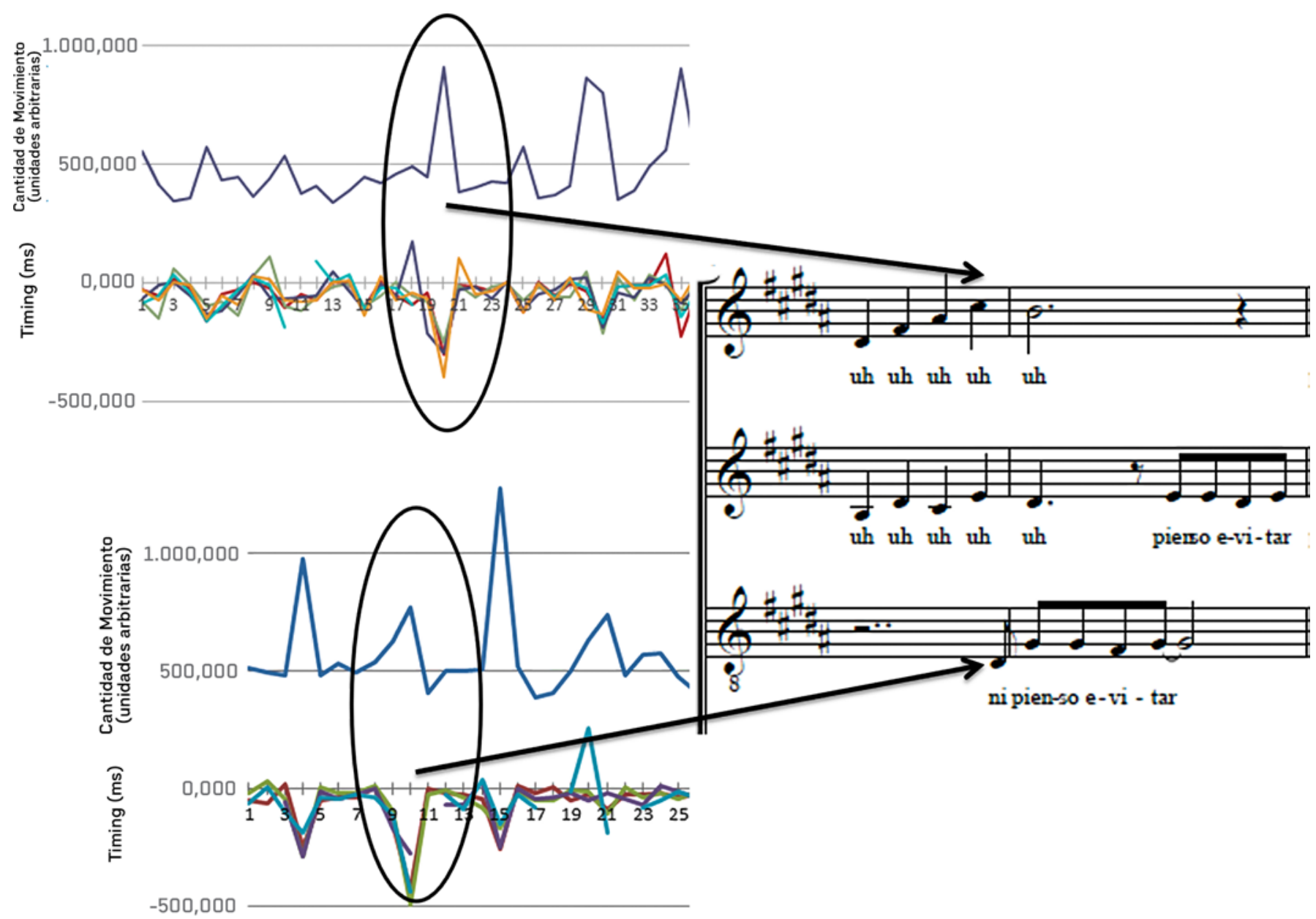

Figura 4. Perfiles de timing de la cuerda de sopranos (izquierda superior) y tenores-bajos (izquierda inferior) en la misma ubicación temporal de la obra (ilustrado en el fragmento de partitura hacia la derecha). La línea azul superior representa la dinámica de movimientos del director.

temporalmente antes del o.20 (es decir, hay un adelantamiento) debido a la doble clave visual que el director comunica con sus manos y cabeza simultáneamente. El director es quien comunica una anticipación a la cuerda masculina, y podría ser lo que también anticipan las sopranos. Es decir, si bien los hombres respondieron como se les indicó, aquí las mujeres respondieron como se les comunicó a los hombres (0.10-11 en los hombres y o.19-20 en las sopranos). El pico en la curva de movimiento anterior en el gráfico que ilustra la ejecución de los varones (Figura 4) es la anticipación del director.

Podríamos decir que las sopranos se anticipan porque ese valor corto y rápido (de corchea) supone una cualidad dinámica que conduce a la caída. Esta es la llegada de las sopranos frente al levare de corchea de los varones. Consultando el video del fragmento ${ }^{2}$ se puede apreciar el doble gesto del director.

2 https://1drv.ms/v/s!Ar1txfVdbUlngeZSbskhlnC-xxelWQ
La cantidad de movimiento del director es una señal al coro para la organización de la performance. Es un indicador de la organización temporal de eventos de diferente naturaleza, tales como (Figura 5):

- entradas (como vemos en la Figura 5 cuando ocurren las entradas de las otras voces),

- inicios de importancia melódica (0.10-11),

- anticipación de los finales formales (0.29-30 donde hay una importante articulación formal del fragmento),

- terminación de frases o rallentandi (o.64-65), y así sucesivamente.

Por lo tanto, en términos de la comunicación dinámica director-coro no son sólo los gestos de dirección, sino el complejo de movimientos del director (toda su corporalidad) que aumenta su presencia en lugares clave de la performance, generando diferentes tipos de respuesta por parte del coro, como los anteriormente descriptos. 


\section{Desvíos de timing (TENOR) y Dinámica de movimiento (DIRECTOR)}

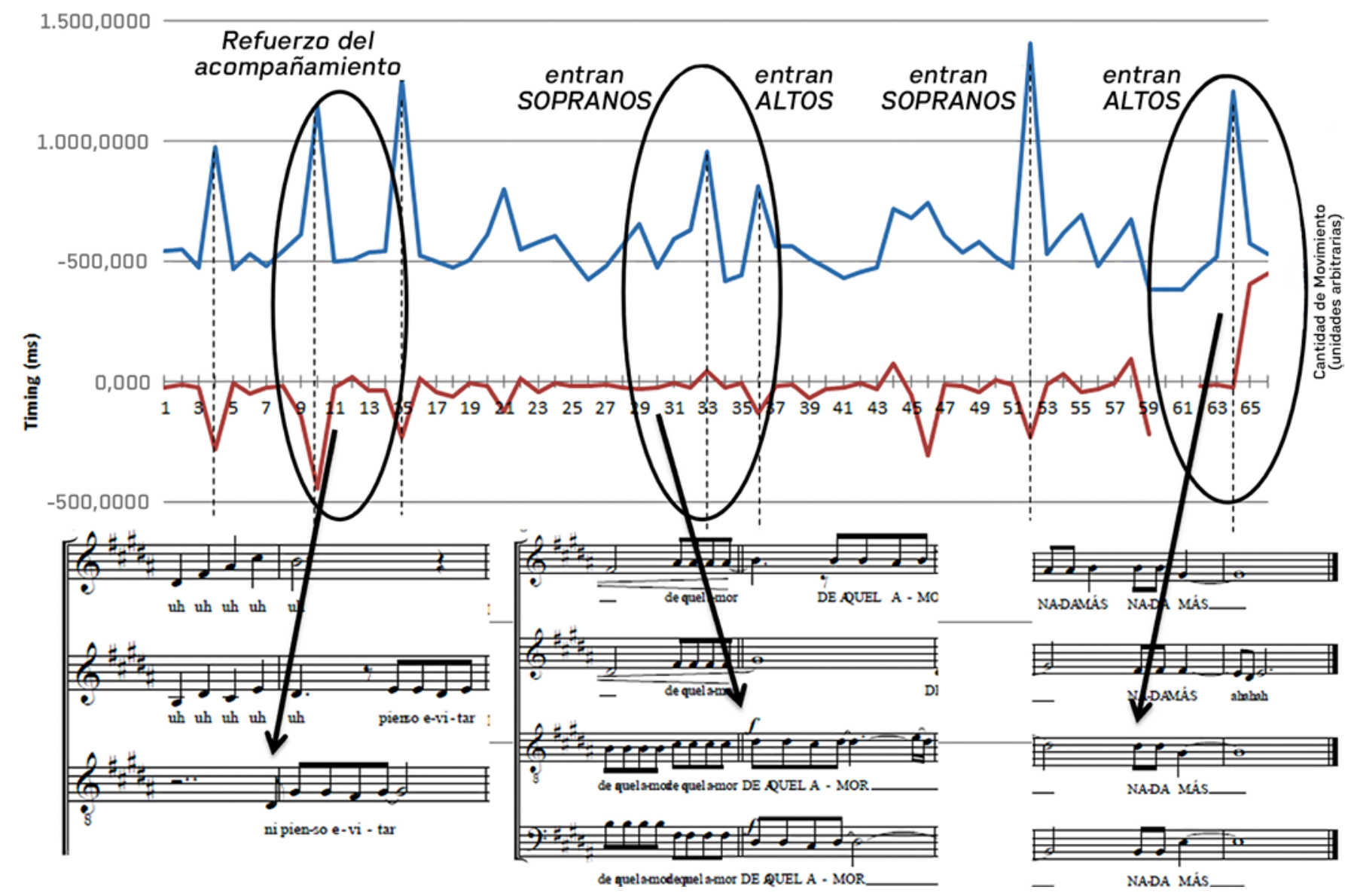

Figura 5. Perfiles de timing de la cuerda de tenor. El eje y muestra los valores de desvíos temporales en milisegundos. El eje $x$ corresponde a las notas cantadas por el tenor. La línea azul superior representa la dinámica de movimientos del director.

En estos análisis, observamos que la aparente homogeneidad —normalmente concebida como el resultado generado por las acciones del director - es de hecho el resultado de un proceso performativo de múltiple vía que oculta una variabilidad interna sonoro-kinética.

\section{Discusión}

Volviendo a la idea de que el director trabaja en busca de la homogeneidad, la construcción de esa homogeneidad consistiría en individualidades heterogéneas que se adaptan gradualmente al comportamiento del grupo, ya sea a la propia cuerda o el canto individual en relación al grupo y al director. La observación en el microanálisis mostró una ligera variabilidad en la definición del ataque de las sopranos. La doble indicación de la anticipación temporal del gesto del director se convirtió en una clave que desvió su atención. Por lo tanto, es factible concluir que la variabilidad del sonido de las sopranos estuvo relacionada con una posible mala interpretación de esa característica visual.
Entendemos que la homogeneidad en la producción y la comunicación es algo que hay que construir. Sería de esperar entonces que, en la práctica de este coro, con el tiempo, la cuerda de sopranos reaccione de forma homogénea a una señal y no siga la otra señal que no le está siendo comunicada. En un futuro ensayo, por ejemplo, se esperaría que el director les diga a las sopranos: "miren las manos y no la cabeza", y a los bajos: "miren la cabeza y no las manos".

Pareciera haber una variabilidad intrínseca de las compensaciones temporales individuales dentro del conjunto coral y entre las cuerdas (como vimos en el timing intra-cuerda de las sopranos) pero también hay algunos casos de variabilidad que se relacionan con la construcción de una comunicación entre el director y el coro, basado en el ajuste de las diferencias en los gestos del director y en las respuestas del coro, progresando hacia la homogeneidad. Así, mientras que el director utiliza un aumento de movimiento para acompañar los puntos estructurales de la forma, las entradas de importancia musical, los cambios de la sección 
formal y las variaciones temporales como los rallentandi, los cantantes por otro lado, muestran mayor o menor variabilidad fundamentalmente con comportamiento compensatorio temporal dentro de la cuerda (expresado en forma de aceleraciones y desaceleraciones temporales durante la ejecución).

Consideramos que estos estudios descriptivos del movimiento interno, el dinamismo y la variabilidad en el coro proporcionan un valioso conocimiento para ser transferido a varias áreas de estudio, así como sistemas de comportamiento en la práctica coral. Hay algunos estudios cualitativos que complementarían de alguna manera a las mediciones y conclusiones aquí obtenidas, en un intento de ampliar esta mirada interactiva de la práctica coral y la dirección musical de grupos vocales. Mediante un análisis cualitativo, Ordás y Martínez (2019b) encontraron que, aunque los coreutas manifiestan que deben sonar como una única entidad, uniforme y donde no se perciban desajustes de ningún tipo, en el análisis hacia el interior del coro, sus relatos reflejan que hay un reconocimiento de la variabilidad en términos de heterogeneidades de todo tipo, vinculada a las claves multimodales, en particular a las claves sonoro-kinéticas. Los participantes del coro hablaban de la propia cuerda y de las otras cuerdas haciendo referencia en su relato al concepto de masa uniforme; sin embargo, también hablaron de la variabilidad interna y del movimiento interno de la cuerda, reconociendo liderazgos dentro de la totalidad que no provienen únicamente del director, sino también que emergen de sus descripciones respecto del volumen y la afinación, por mencionar algunas. Los resultados de dicho estudio también informaron acerca de la idea de construcción conjunta de significados. En este sentido, podemos decir que, sobre esa construcción, que es de por sí muy diversa, se constituye la búsqueda de la homogeneidad. Dicho de otro modo, se construye la sonoridad conjunta a partir de la diversidad; en base a nuestros resultados, esta última no debería ser valorada en términos negativos, como suele ocurrir en la práctica coral tradicional, sino como el componente sobre el cual se construye el sonido vocal grupal.

Sostenemos, sin embargo, que debe seguir profundizándose en futuras investigaciones con propuestas multi-método, esto es, mediante estudios mixtos que empleen métodos tanto cuantitativos como cualitativos para lograr una mayor consistencia en la formulación de hipótesis y discutir los estudios relativos a la experiencia musical, que, por su naturaleza, son intrínsecamente multimodales. Mediante el empleo de diversos enfoques empíricos, proponemos indagar la actividad en el coro mediante la búsqueda de indicios relativos al comportamiento multimodal. Entendemos el coro como un conjunto de subjetividades que construyen el significado de su práctica en función de claves visuales, auditivas, espaciales y temporales. Para comprender la experiencia del coro postulamos que operan estas claves - multimodales - que se modifican y transforman; es por ello que incluimos la intersubjetividad en el análisis, por lo que se amplía el campo disciplinar.

Este estudio es una primera mirada. Una mayor investigación implicaría el desarrollo de un modelo de comunicación en el marco de la construcción de la temporalidad. Lejos de pretender erigirse como una única verdad universal de los aspectos que describen y forman parte de la práctica, proponemos que el análisis de la práctica situada y limitada al contexto brinda una metodología de abordaje del problema novedosa. Dialogar con los distintos modos de profundizar en nuestro objeto de estudio desde una perspectiva interdisciplinaria, nos permite revisar nuestras concepciones con teorías que postulan la evolución del sentido de pertenencia grupal como una de las características adaptativas humanas que, postulamos, definen a la actividad coral. Luego, estos marcos se amplían con la inclusión de nuevas posturas acerca de la definición del coro que permiten configurar una nueva mirada de la producción y realización en la práctica coral. Asimismo, abogamos a que la investigación futura contribuya a la pedagogía de la dirección coral reformulando su ontología, sin silenciar la individualidad y entendiendo la variabilidad como una construcción del sonido coral.

\section{Referencias}

Cannam, C., Landone, C., y Sandler, M. (2010). Sonic Visualiser: An Open Source Application for Viewing, Analysing, and Annotating Music Audio Files. En Proceedings of the 18th ACM international conference on Multimedia (MM '10), (pp. 1467-1468). Association for Computing Machinery, New York, NY, USA. doi: https://doi.org/10.1145/1873951.1874248

Clayton, M. (2012). What is Entrainment? Definition and applications in musical research. Empirical Musicology Review, 7(1), 49-56. doi: https://doi. org/10.18061/1811/52979

Clayton, M. (2013). Entrainment, Ethnography and Musical Interaction. En M. Clayton, B. Dueck, y L. Leante 
(Eds.), Experience and Meaning in Music Performance (pp. 188-207). New York: Oxford University Press. doi: 10.1093/acprof:o so/9780199811328.003.0002

Clayton, M., Sager, R., y Will, U. (2005). In time with the music: The concept of entrainment and its significance for ethnomusicology. European Meetings in Ethnomusicology 11 (ESEM Counterpoint 1), 1-82.

Damesón, J. A. (2018). Gestos en la dirección: Comunicación de significados musicales corporeizados. Tesis de maestría, Facultad de Artes, Universidad Nacional de La Plata. La Plata. doi: https://doi. org/10.35537/10915/67993

Loehr, J. D. y Palmer, C. (2011). Temporal Coordination between performing musicians. The Quarterly journal of experimental psychology, 64(11), 2153-2167. doi: https://psycnet.apa.org/doi/10.1080/17470218.2011.60 3427

Martínez, I. C., Dameson, J., Pereira Ghiena, A., Tanco, M., y Alimenti Bel, D. (2017). Participatory Sense Making in Jazz Performance: Agents' Expressive Alignment. ESCOM 2017. Expressive Interaction with Music. 25th Anniversary Edition of the European Society for the Cognitive Sciences of Music (ESCOM). University of Ghent. Bélgica.

Ordás, M. A. (2017). La comunicación intersubjetiva en la práctica del coro. Claves multimodales e interacción entre los coreutas y el director. Tesis doctoral, Facultad de Artes, Universidad Nacional de La Plata. La Plata. doi: https://doi.org/10.35537/10915/61687

Ordás, M. A. (2018). Variabilidad temporal hacia el interior del coro: Un estudio de claves multimodales y timing expresivo en la práctica coral. En M. A. Ordás, M. Tanco e I. C. Martínez (Eds.), Experiencia, producción y pensamiento. Libro de resúmenes de las Jornadas de Investigación en Música, (pp. 87-89). La Plata: Laboratorio para el Estudio de la Experiencia Musical (LEEM), Facultad de Bellas Artes, Universidad Nacional de La Plata.

Ordás, M. A. y Blanco Fernández, M. A. (2013). La corporeidad y la expresión en la ejecución coral. Indicios de la identificación del cuerpo como productor de mensajes. En F. Shifres, P. Jaquier, D. Gonnet, M. I. Burcet, y R. Herrera (Eds.) Nuestro Cuerpo en Nuestra Música, Actas de 11o ECCoM, 1(2), (pp. 455-465). Buenos Aires: SACCoM.
Ordás, M. A. y Martínez, I. C. (2013). Incidencia de las fuentes de información temporal en la inducción del beat durante la recepción multimodal de una ejecución coral. En F. Shifres, P. Jacquier, D. Gonnet, M. I. Burcet, y R. Herrera (Eds.) Nuestro Cuerpo en Nuestra Música, Actas de 11ํㅡㄹ ECoM, 1(2), (pp. 467-478). Buenos Aires: SACCOM.

Ordás, M. A. y Martínez, I. C. (2017). La actividad temporal en la cuerda del coro. Un estudio de timing expresivo. En N. Alessandroni y M. I. Burcet (Eds.), La experiencia musical: abordajes desde la investigación, la interpretación y las prácticas educativas. Libro de resúmenes del 13.ํㅡㄹ Encuentro de Ciencias Cognitivas de la Música (pp. 65-66). Buenos Aires: SACCoM.

Ordás, M. A. y Martínez, I. C. (2019a). Claves multimodales en la comunicación intersubjetiva del Coro. En N. Alessandroni, B. Torres Gallardo y C. Beltramone (Eds.). Vocalidades: la voz humana desde la interdisciplina (pp. 367-417). La Plata: GITeV.

Ordás, M. A. y Martínez, I. C. (2019b). La variabilidad como construcción de significado en el coro. Epistemus. Revista de Estudios en Música, Cognición y Cultura, 7(2), 010. doi: https://doi. org/10.24215/18530494e010

Ordás, M. A., Martínez, I. C., y Alimenti Bel, D. (2014). Claves Visuales en la Dirección Coral: Variabilidad gestual en la comunicación de patrones temporales. En M. Escobar y L. Gasparoni (Eds.) Música Latinoamericana: Tradición e innovación. Actas de las III Jornadas de la Escuela de Música de la U.N.R. Rosario: UNR Editora.

Pazniakova, L., Maes, P.-J., Desmet, F., y Leman, M. (2016). Understanding the Conductor's Communication of Expressive Timings - the Role of Tempo, Auditoryvisual Modality, Musical Expertise and Movement. Recuperado de https://www.researchgate.net/ publication/310672967

Repp B. H. (2000). Subliminal temporal discrimination revealed in sensorimotor coordination. En P. Desain, y L. Windsor (Eds.). Rhythm Perception and Production (pp. 129-142). Lisse: Swets \& Zeitlinger.

Repp B. H. (2002). Phase correction following a perturbation in sensorimotor synchronization depends on sensory information. Journal of Motor Behavior, 34(3), 291-298. doi: 10.1080/00222890209601947 
Repp B. H. (2005). Sensorimotor synchronization: A review of the tapping literature. Psychonomic Bulletin \& Review, 12, 969-992. doi: https://doi.org/10.3758/ BF03206433

Repp B. H. y Doggett R. (2007). Tapping to a very slow beat: A comparison of musicians and non-musicians. Music Perception, 24(4), 367-376. doi: https://doi. org/10.1525/mp.2007.24.4.367

Repp B. H. y Keller P. E. (2004). Adaptation to tempo changes in sensorimotor synchronization: Effects of intention, attention, and awareness. The Quarterly Journal of Experimental Psychology Section A, 57(3), 499-521. doi: 10.1080/02724980343000369

Repp B. H. y Su YH. (2013). Sensorimotor synchronization: A review of recent research (20062012). Psychon. Bull. Rev., 20, 403-452. doi: https://doi. org/10.3758/s13423-012-0371-2

Repp, B. H., y Penel, A. (2002). Auditory dominance in temporal processing: New evidence from synchronization with simultaneous visual and auditory sequences. Journal of Experimental Psychology: Human Perception and Performance, 28(5), 1085-1099. doi: https://doi.org/10.1037/0096-1523.28.5.1085

Schiavio, A. (2014). Action, Enaction, Inter(en)action. Empirical Musicology Review, 9(3-4), 254-262. doi: http://dx.doi.org/10.18061/emr.v9i3-4.4440

Schiavio, A. y De Jaegher, H. (2017) Participatory SenseMaking in Joint Musical Practice. En M. Lesaffre, M. Leman, y P. J. Maes (Eds), The Routledge Companion to Embodied Music Interaction, (pp. 31-139). New York: Routledge.

Schiavio, A. y Høffding, S. (2015). Playing together without communicating? A pre-reflective and enactive account of joint musical performance. Musicae Scientiae, 19(4), 366- 388. doi: https://doi. org/10.1177/1029864915593333

Timmers, R., Endo, S., Bradbury, A., y Wing, A. M. (2014). Synchronization and Leadership in String Quartet Performance: a case Study of Auditory and Visual Cues. Frontiers in Psychology, 5(1), 645-654. doi: 10.3389/ fpsyg.2014.00645.
Wesolowski, B. C. (2016). Timing Deviations in Jazz Performance: The Relationships of Selected Musical Variables on Horizontal and Vertical Timing Relations: A Case Study. Psychology of Music, 44(1), 75-94. doi: 10.1177/0305735614555790. 